\title{
KN07 | Chemistry And Crystallography At Ultra-High Pressures
}

Bykova, Elena (Bayerisches Geoinstitut, Bayreuth, GER)

Many scientific disciplines including geophysics, geochemistry, mineralogy, material sciences and engineering are interested in study of materials exposed to extreme conditions as high pressures and high temperatures (HPHT). External stimuli can trigger structural, electronic and magnetic changes in a matter; chemical reactions conducted at HPHT can demonstrate unexpected behavior totally different from that at ambient conditions. However, the analysis and interpretation of the data collected at multimegabar pressures, especially after/during laser heating, is extremely difficult due to unknown (and often very exotic, not known at ambient conditions) chemistry of the materials. Single-crystal X-ray diffraction in diamond anvil cells provides a unique opportunity to study in-situ such important characteristic of a material as its crystal structure (unit cell parameters, a space group, atomic coordinates and atomic occupancies), and, if required, a phase composition. Here we demonstrate a great potential and novel opportunities provided by high-pressure crystallography in materials- and geo-sciences on the example of studies of iron oxides, silica and carbonates. 